\title{
A Fault-Cored Anticline Boundary Element Model Incorporating the Combined Fault Slip and Buckling Mechanisms
}

\author{
Wen-Jeng Huang ${ }^{1, *}$ and Kaj M. Johnson ${ }^{2}$ \\ ${ }^{1}$ Graduate Institute of Applied Geology, National Central University, Taoyuan, Taiwan, R.O.C. \\ ${ }^{2}$ Department of Geological Sciences, Indiana University, Indiana, U.S.A.
}

Received 30 September 2014, revised 12 April 2015, accepted 18 June 2015

\begin{abstract}
We develop a folding boundary element model in a medium containing a fault and elastic layers to show that anticlines growing over slipping reverse faults can be significantly amplified by mechanical layering buckling under horizontal shortening. Previous studies suggested that folds over blind reverse faults grow primarily during deformation increments associated with slips on the fault during and immediately after earthquakes. Under this assumption, the potential for earthquakes on blind faults can be determined directly from fold geometry because the amount of slip on the fault can be estimated directly from the fold geometry using the solution for a dislocation in an elastic half-space. Studies that assume folds grown solely by slip on a fault may therefore significantly overestimate fault slip. Our boundary element technique demonstrates that the fold amplitude produced in a medium containing a fault and elastic layers with free slip and subjected to layer-parallel shortening can grow to more than twice the fold amplitude produced in homogeneous media without mechanical layering under the same amount of shortening. In addition, the fold wavelengths produced by the combined fault slip and buckling mechanisms may be narrower than folds produced by fault slip in an elastic half space by a factor of two. We also show that subsurface fold geometry of the Kettleman Hills Anticline in Central California inferred from seismic reflection image is consistent with a model that incorporates layer buckling over a dipping, blind reverse fault and the coseismic uplift pattern produced during a 1985 earthquake centered over the anticline forelimb is predicted by the model.
\end{abstract}

Key words: Folding, Earthquake hazard, Anticline, Fault-related fold, Blind fault, Fault slip rate

Citation: Huang, W. J. and K. M. Johnson, 2016: A fault-cored anticline boundary element model incorporating the combined fault slip and buckling mechanisms. Terr. Atmos. Ocean. Sci., 27, 73-85, doi: 10.3319/TAO.2015.06.18.01(TT)

\section{INTRODUCTION}

Geodetic observations have been interpreted as evidence that anticlines over blind faults grow as a consequence of slip on faults during earthquakes. King and Stein (1983), Stein and King (1984), and Stein and Ekström (1992) proposed that a string of Quaternary folds in Central California at Coalinga and Kettleman Hills are produced by sudden, incremental growth during repeated earthquakes on underlying blind reverse faults (Fig. 1). This idea is leveraged by observations at Coalinga Anticline that show a similarity in pattern between uplifted river terraces, current topography and coseismic vertical displacements determined from leveling measurements before and after the 1983 Coalinga earthquake. Stein and Ekström (1992) inferred a fault slip rate for

\footnotetext{
* Corresponding author

E-mail:huang22@ncu.edu.tw
}

the blind fault underlying Coalinga assuming that the uplift rate of the fold directly reflects the slip rate on the fault.

This view that anticlines grow primarily by slip on the underlying fault is reflected in other fault-related folding studies (e.g., Taboada et al. 1993; Myers et al. 2003; Mynatt et al. 2007). In addition, a growing number of studies attempt to link active anticline growth with slip on an underlying fault. The approach in these studies is to determine the geometry and uplift rate of active fault-related folds and then infer the fault slip rate from an assumed kinematic relationship between the fault slip and fold shape (e.g., Grant et al. 1999; Shaw and Shearer 1999; Allmendinger and Shaw 2000; Shaw et al. 2002; Ishiyama et al. 2004; Le Béon et al. 2014).

The main message of this paper is that the geometry of anticlines produced solely by slip on underlying reverse 
faults is significantly different from the geometry of anticlines produced by the combined fault slip and buckling mechanisms of mechanical layers under horizontal shortening. Layer buckling has the effect of amplifying and narrowing the fold produced by slip on the underlying fault. To date, mechanical analyses of fault-related folding have largely ignored the buckling process because either the mechanical layers required for buckle folding are absent in such analyses or passive layer folding due to slip on a fault is considered without horizontal shortening. Perhaps the influence of buckling in fault-related folds has been largely ignored because previous studies on the mechanics of buckle folding have focused on the formation of repetitive fold forms in layered media (e.g., Johnson and Fletcher 1994), which are rarely observed in sedimentary rocks. Yet, field observations (e.g., Erslev and Mayborn 1997) show that layer-parallel slip appears ubiquitously in fault-cored anticlines, and therefore the role of buckling accommodated by slip at layer contacts should not be neglected in faultrelated folding analyses.

We develop a boundary element model of the growth of an anticline over a fault embedded in a medium with elastic layers that slip at the contacts. We demonstrate that fault-cored folds in a mechanically layered medium can be significantly amplified and localized by buckling under horizontal compression. To demonstrate this point, we examine the subsurface geometry and surface deformation measurements from the active Kettleman Hills and Coalinga Anticlines in Central California.

\section{KINEMATICS AND MECHANICS OF FAULT-CORED ANTICLINES}

In this paper, we focus our attention on anticlines that form above buried reverse faults. Three classes of faultrelated folds that are most relevant to this particular geometry are fault-bend folds, fault-tip/fault-propagation folds and forced-folds. Fault-bend folds form when rock moves through a flat-ramp-flat fault geometry and generates repetition of the section and a ramp anticline (Rich 1934; Suppe 1983). Fault propagation and fault-tip folds form by shortening and shear generated at the terminations of propagating or non-propagating reverse faults. Forced folds form in a medium overlying displaced rigid basement blocks. A number of kinematic models, similar to the Suppe (1983) faultbend-fold model with straight limbs and sharp hinges, have been constructed to capture the basic geometry of fault-tip and fault-propagation folds (e.g., Chester and Chester 1990; Suppe and Medwedeff 1994). The trishear kinematic model (e.g., Erslev 1991; Cardozo 2008) is particularly popular in the recent literature because it produces rounded fold forms that look more like natural folds and has been used to model forced-folds and fault-tip folds.

Theoretical models investigating the passive folding of markers in various rheology materials in response to slip on a underlying fault have been developed by a number of researchers [models for ramp folding (e.g., Elliot 1976; Wiltschko 1979; Berger and Johnson 1980, 1982; Johnson and Berger 1989; Erickson and Jamison 1995; Strayer and Suppe 2002); models for forced folds (e.g., Sanford 1959; Reches and Johnson 1978; Patton and Fletcher 1995; Johnson and Johnson 2002; Cardozo et al. 2003, 2005; Finch et al. 2003); models for fault propagation folding (e.g., Cardozo et al. 2005)]. To our knowledge few studies have incorporated buckling, mainly interlayer slip. Boundary element models were developed by Cooke and Pollard (1997) and Shackleton and Cooke (2007) to analyze the contribution of frictional slip along bedding planes to fault-related folding of layered rocks. The former mainly investigated the deformation of frictional bedding planes near dipping faults under layer-parallel contraction and extension. The latter focused on evaluating the validity of the plane strain assumption in non-cylindrical folds. Finite element models were developed by Niño et al. (1998) to analyze the propagation of a blind thrust in a deformable basement in terms of the evolution of strain localization in the overlying elastoplastic layers; studying the layer thickness, bedding-parallel slip and fault dip roles. In addition, Smart et al. (2009) used finite-elementbased geomechanical models of fault related folds to show the impact of interlayer slip on fracture prediction.

While little attention has been afforded to the mechanics of fault-related fold buckling, the theory of folding of initial perturbations in isolated layers or multilayers without faulting is quite mature (e.g., Biot 1963, 1964; Chapple 1969; Fletcher 1977; Johnson 1977; Kilsdonk and Fletcher 1989; Johnson and Fletcher 1994; Mancktelow 1999). Of particular relevance to this paper are theoretical studies on the physical conditions of multilayer folding that lead to significant amplification of initially small perturbations. In linear, homogenous materials, the rate at which an initial perturbation is amplified is a function of the number of layers in the multilayer, $N$, the thickness of the individual layers, $h$, and the wavelength, $L$, of the initial perturbation. The rate at which initial sinusoidal perturbations are amplified by buckling under horizontal compression was quantified by Biot (1961) and Fletcher (1977) as the "amplification factor". The amplification factor is a scalar quantity that determines the rate at which the amplitude of an initially small perturbation grows with increased shortening of the medium (e.g., Johnson and Fletcher 1994).

Figure 2, produced from the folding theory developed by Johnson and Pfaff (1989), shows the amplification factor as a function of the perturbation wavelength normalized by the thickness of a single layer. The layers have viscosity equal to the surrounding media and free slip at layer contacts. The amplification factor is shown for multilayers with two, four, or ten layers. Figure 2 illustrates that the amplification factor (i.e., the rate at which the amplitude grows) increases 
with the number of layers in the multilayer. For a given layer thickness and number of layers, a so-called dominant wavelength exists at which the amplification factor is largest and the fold grows the fastest (the peak of the curves). The amplification factor concept is relevant in a general way to fault-cored folding in a multilayer under layer-parallel shortening. In this case, we expect the length of the perturbation produced by slip on the fault would be controlled by the fault geometry. We would expect the rate of growth of the faultcored fold to be a function of the shortening rate, the rate of slip on the fault, and the layer thickness and number of layers. A fault-cored fold in a medium with no mechanical layering is expected to grow more slowly than a fold overlying a fault in a medium with many mechanical layers.

\section{BOUNDARY ELEMENT MODEL OF FAULT-RELATED FOLDING}

We develop a boundary element model to examine the fault-related fold amplification by buckling. The boundary element method (BEM) is different from the finite element method (FEM) in that the medium is discretized only at boundaries in the BEM whereas the entire medium is discretized in the FEM.

\subsection{Basic Formulation}

In layered sedimentary rocks mechanical interfaces between sedimentary layers may form because of differences

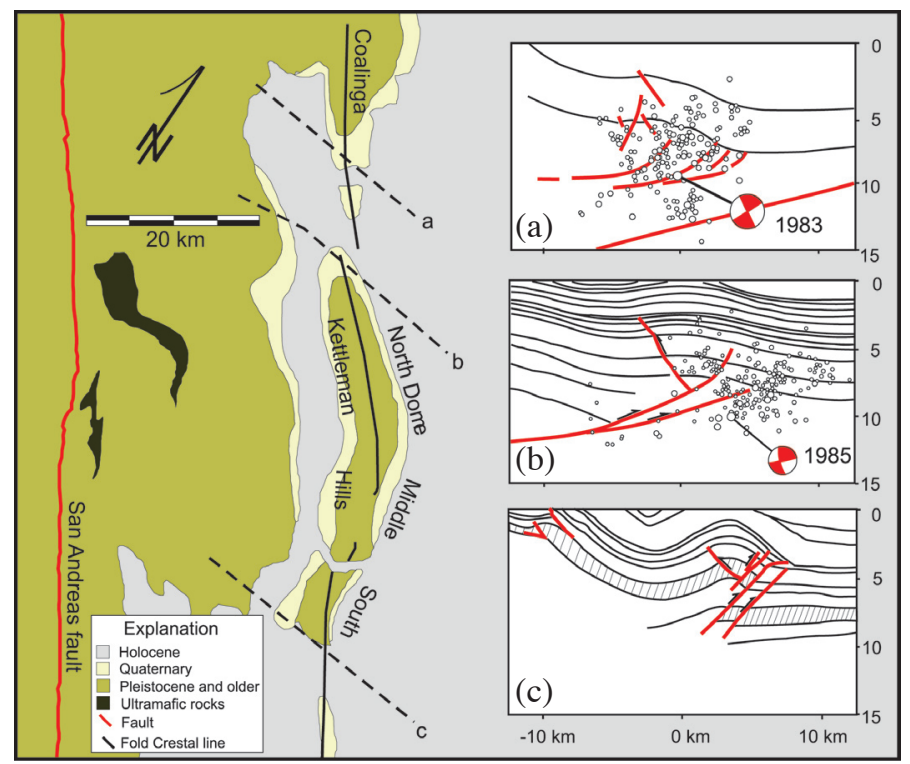

Fig. 1. Geological map and cross sections of Coalinga and Kettleman Hills (modified after Stein and Ekström 1992). Geometry is based on seismic reflection images. (a) Coalinga profile with moment tensor for 1983 earthquake. Hypocenters of small earthquakes are shown with small circles. (b) Kettleman Hills North Dome with moment tensor for 1985 earthquake. Hypocenters of small earthquakes are shown with small circles. (c) Kettleman Hills South Dome.
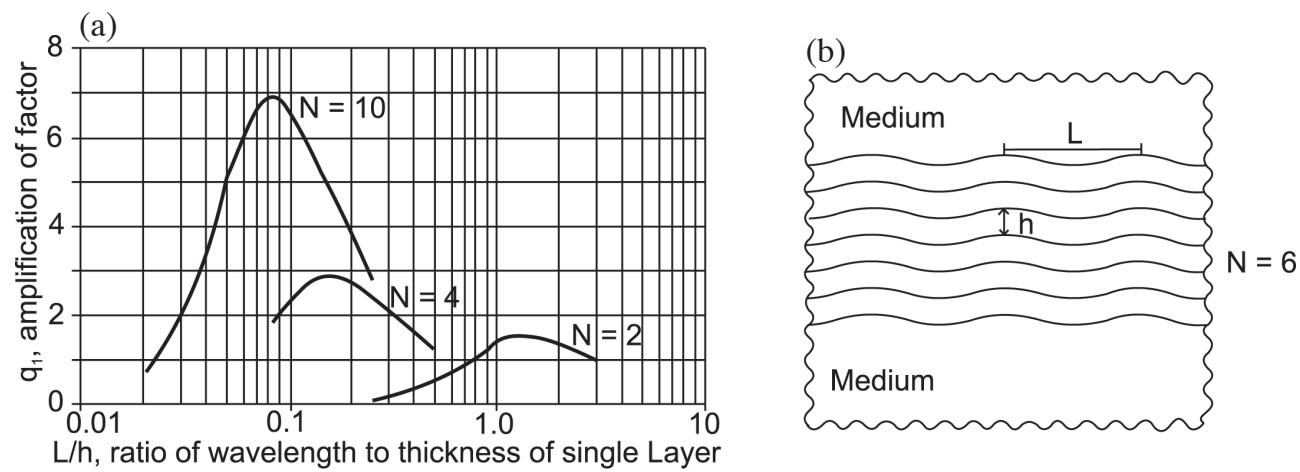

Fig. 2. (a) Plots of amplification factor for periodic folds in viscous layers as a function of wavelength, $L$, normalized by the thickness of a single layer, $h . N$ indicates number of layers in multilayer. Layers slip freely at contacts. Layers and surrounding medium have same viscosity. The plots are produced using the folding theory developed by Johnson and Pfaff (1989). (b) Illustration of a multilayer bounded above and below by semiinfinite media. The number of layers in the multilayer, $N$, is 6 . 
in physical properties at the interfaces such as grain size and cementation. Soft layers interbedded with stiff layers may localize shear, allowing the stiff layers to slide past each other. These conditions are important in folding because the bedding-plane slip can allow the strata to mechanically buckle with flexural slip. We model these conditions with multiple elastic layers with frictional contacts (Fig. 3).

The basic geometry and boundary conditions of two classes of models are illustrated in Figs. 3a and b. We model mechanical layers with initially horizontal slip surfaces of finite length within an otherwise homogeneous elastic halfspace. The fault may be embedded in the layers or below the layers in the half-space. In general the layers and the fault are assumed to slip according to a Coulomb friction law, $\left|\tau_{s}\right| \leq C+\mu \sigma_{n}$, where $\tau_{s}$ is shear stress, $C$ is cohesion, $\mu$ is the coefficient of friction and $\sigma_{n}$ is normal stress (compression is positive). The entire medium is subjected to increments of either uniform strain (Fig. 3a) or uniform displacement above the detachment (Fig. 3b). If the shear stress on the fault or layers exceeds the strength as defined by the Coulomb friction law during each increment of applied displacement or far-field strain the interfaces slip in order to reduce the shear stress to the strength. In this paper we restrict our attention to the frictionless, cohesionless case, $C=\mu=0$, because this special case simplifies the illustration of the effect of mechanical layering on fold growth.

The BEM numerical technique has been clearly described by Crouch and Starfield (1983). Our boundary element algorithm is largely similar to their two-dimensional displacement discontinuity method (TWODD) which was succinctly summarized by Martel and Muller (2000). We formulate the elastic boundary element models using the solution for an edge dislocation in an isotropic, homogeneous, elastic half-space assuming infinitely long faults and bedding contacts in the strike direction (2D plane-strain conditions).

We give a brief outline of our formulation of the bound- ary element model. Assume we have a $N \times 1$ vector of incremental values of the dip component of slip, $s$, on $N$ patches. From the solution for a $2 \mathrm{D}$ edge dislocation, we can relate the vector of shear stresses, $\sigma_{s}$, at the center of each patch to slip on all the patches through the $N \times N$ matrix, $G_{\sigma}$,

$\sigma_{s}=G_{\sigma} s$

We assume a coordinate system with $x$ in the horizontal direction and $y$ in the vertical direction. We apply increments of far-field uniform strain,

${ }^{f f} \varepsilon_{x x}=$ constant, ${ }^{f f} \varepsilon_{y y}=-{ }^{f f} \varepsilon_{x x} \nu /(1-\nu),{ }^{f f} \varepsilon_{x y}=0$

with corresponding uniform far-field stress,

${ }^{f f} \sigma_{y y}={ }^{f f} \sigma_{x y}=0,{ }^{f f} \sigma_{x x}=2 \mu{ }^{f f} \varepsilon_{x x}+\lambda\left({ }^{f f} \varepsilon_{x x}+{ }^{f f} \varepsilon_{y y}\right)$

where $\lambda$ and $\mu$ are Lame's elastic constants, $\lambda=2 \mu \nu /(1-2 v)$, and $\nu$ is Poisson's ratio. We normalize all stresses using $\mu$ and assume $v=0.25$. From the far-field stress we compute the shear component of stress resolved onto each patch, ${ }^{f f} \sigma_{s}$. Assuming cohesionless, frictionless contacts, we satisfy the condition that the shear stress is zero on each patch after each increment of deformation,

${ }^{f f} \sigma_{s}+G_{\sigma} s=0$

The distribution of incremental slip on the patches that gives this condition is

$s=G_{\sigma}^{-1}\left(-{ }^{f f} \sigma_{s}\right)$

$N \times N$ matrices ${ }_{x} G_{d}$ and ${ }_{y} G_{d}$ relating the $x$ and $y$ components (a)

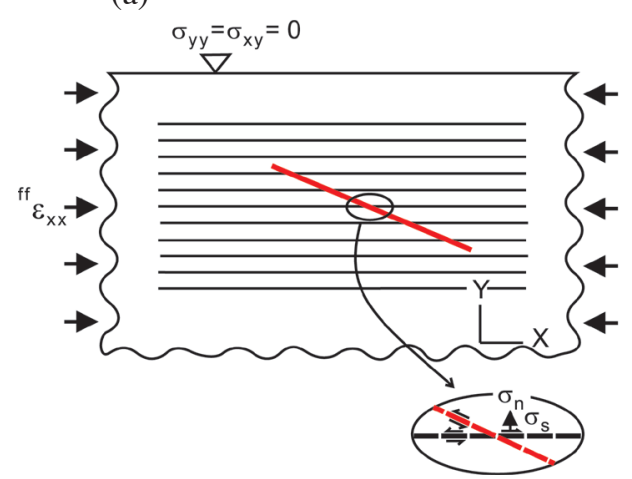

(b)

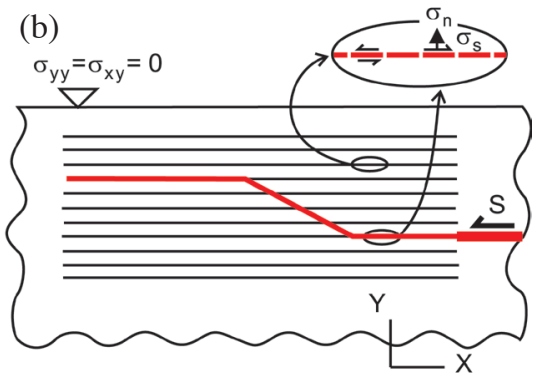

Fig. 3. Geometry and boundary conditions of two models of a fault embedded in an elastic medium with mechanical layering. Notation is $\sigma$ : stress, $\sigma_{n}$ : normal traction, $\sigma_{s}$ : shear traction, ${ }^{f f} \varepsilon$ : remote strain, and $S$ : uniform slip. Wiggly edges indicate that the medium extends to infinity. (a) Embedded fault case. The loading condition is horizontal shortening, i.e., ${ }^{f f} \varepsilon_{x x}$. (b) Ramp fault case. The loading condition is a uniform slip applied to the detachment on the far right side of the model domain. (Color online only) 
of displacements of the endpoints of the patches to the slip on each patch is constructed using the solution for the edge dislocation. Note that we only specify one boundary condition and solve for only one slip component on each patch because we assume that the normal component of displacement discontinuity across patches is zero. The incremental displacements, $\Delta u_{x}$ and $\Delta u_{y}$, of the patch endpoints during the small deformation increment are then calculated as

$$
\Delta u_{x}={ }_{x} G_{d} s+{ }^{f f} \Delta u_{x}, \Delta u_{y}={ }_{y} G_{d} s+{ }^{f f} \Delta u_{y}
$$

with the contribution to the displacements from the far-field strain being

$$
{ }^{f f} \Delta u_{x}=x^{f f} \varepsilon_{x x},{ }^{f f} \Delta u_{y}=y^{f f} \varepsilon_{y y}
$$

New patch endpoint positions are calculated from the previous endpoints and the incremental displacements. A new far-field strain increment is then applied and the calculations in Eqs. (1) - (7) are repeated. For each far-field strain increment we fix the $y$-coordinate of the coordinate system origin at the ground surface (free surface). Note that because we assume zero resistance to sliding on the faults and layer interfaces, we do not need to consider confining pressure due to the lithostatic load. Also for simplicity we ignore the topography build-up and assume that erosion and deposition maintain a flat and horizontal ground surface. Any points on the interfaces that move above the ground surface are discarded.

It is important to recognize that we have adopted the linear (infinitesimal strain) elastic solution for an edge dislocation, yet we do not restrict our analysis to small strains. We assume that each deformation increment can be modeled with the small strain theory, ignoring nonlinear effects due to the initial stress condition at the beginning of each increment. This is equivalent to assuming that the elastic stresses in the medium surrounding the faults and layer interfaces are somehow relaxed before the beginning of the next deformation increment. Inelastic processes for relaxing stresses include: micro-cracking (e.g., Meglis et al. 1995), grain boundary sliding (e.g., Langdon 1970), twinning (e.g., Yamashita and Ojima 1968), pressure solution (e.g., McClay 1977), recrystallization, and so on (Sibson 1986). Because we do not account for these processes in our model, results from this analysis must be viewed with mindfulness of the assumptions. Furthermore, we assume an incremental far-field strain of ${ }^{f f} \varepsilon_{x x}=-0.02$ in all applications in this paper which is about an order of magnitude larger strain than permissible using linear elasticity theory. However, we examine the incremental far-field strain effect with a range of magnitudes between 0.005 and 0.2 on the final fold form. We find that deformation increments equal to or smaller than 0.02 do not produce an appreciable difference in the final fold form indicating that our choice of incremental farfield strain is not a severe limitation.

\subsection{Simulations}

We now show fold forms produced with different fault geometries. For each simulation, we compare folds produced in mechanical layering with folds produced in passive markers with no mechanical layering. We refer to the mechanical layering folds as fault- or ramp-cored buckle folds, and we refer to passive marker folds as fault- or rampcored passive folds.

\subsubsection{Fault in Basement Underlying Sedimentary Layering}

The setting for this case is a fault in a massive rock unit (basement) underlying layers of sedimentary rocks. We model this scenario with a fault embedded in an elastic halfspace underlying a stack of elastic layers. The fault initially dips $25^{\circ}$. Figure 4 shows the results for two extreme conditions for the layer interfaces: bonded (interfaces are passive markers) or freely sliding. We show three stages of folding with maximum fault slip of $0 \mathrm{~W}, 0.14 \mathrm{~W}$, and $0.30 \mathrm{~W}$, where $W$ is the initial fault width (down-dip distance).

The distinct differences between the bonded and freely sliding layers are as follows: (1) the amplitudes of folded interfaces of freely sliding layers grow faster than the amplitudes of the folded passive markers. (2) After maximum fault slip of $0.30 \mathrm{~W}$, the fold in the mechanical layer model is nearly symmetric with tight hinges and curved limbs while the fold in the passive markers is broader. (3) The fold wavelength, measured as the distance between synclinal hinges on the flanks of the anticline, is shorter in the folded mechanical layers than in the folded passive markers. (4) The ratio of average amplitude of folded interfaces to maximum slip on the fault in the fault-cored buckle fold is 1.1 while the ratio for the fault-cored passive fold is 0.23 . Therefore, given the same amount of slip on the fault, the fold amplitude in the fault-cored buckle fold is about 5 times the amplitude of the fault-cored passive fold.

\subsubsection{Fault Embedded in Layers}

Figure 5 shows a model in which an originally straight fault is embedded in the layering. The fault initially dips $25^{\circ}$. We show three stages with maximum fault slip of $0 \mathrm{~W}$, $0.18 \mathrm{~W}$, and $0.30 \mathrm{~W}$. The fault-cored buckle fold form is different from the previous case with the fault below the layers. The crest of the anticline of the fault-cored buckle fold in Fig. 5 forms over the midpoint of the underlying fault, rather than above the fault tip as in the fault-cored buckle fold in the previous model with the fault below the layers and in the fault-cored passive folds in Figs. 4 and 5. The distinct 
(a) Fault-cored passive folding

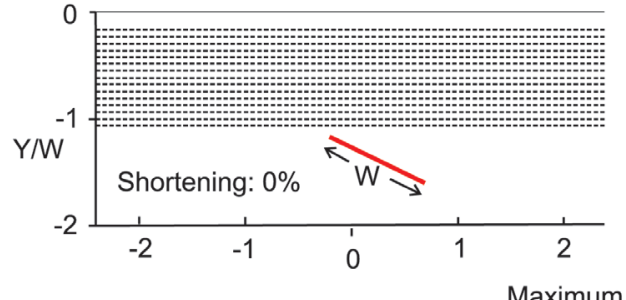

Maximum fault slip: $0.14 \mathrm{~W}$
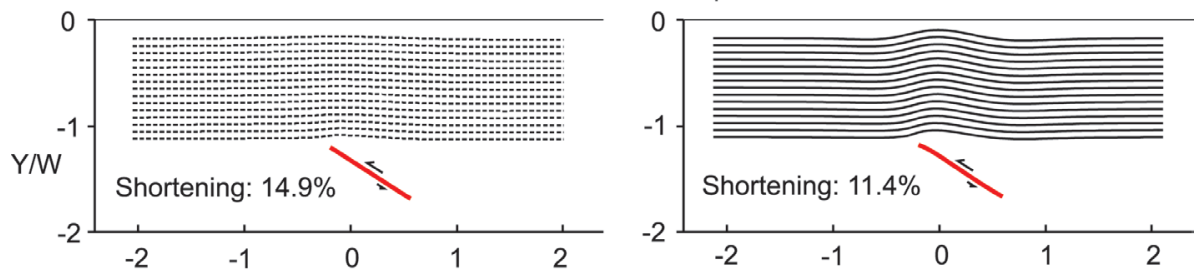

Maximum fault slip: $0.30 \mathrm{~W}$
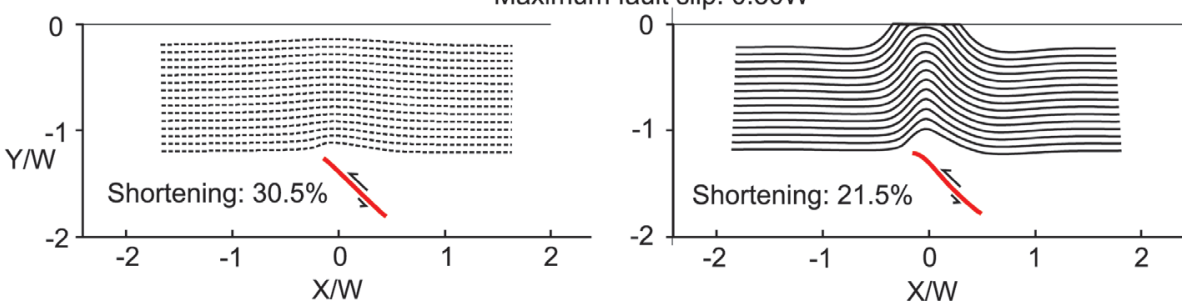

Fig. 4. Models of fault in an elastic half space underlying a stack of elastic layers. Entire medium is subjected to far-field horizontal shortening. $W$ is initial fault width. Fault dips $25^{\circ}$ at onset. (a) Fault-cored passive fold (layers are passive markers.) (b) Fault-cored buckle fold (layers slip freely at contacts). (Color online only)

\section{(a) Fault-cored passive folding}

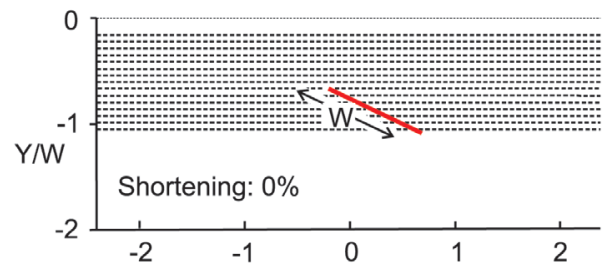

Maximum fault slip: $0.18 \mathrm{~W}$
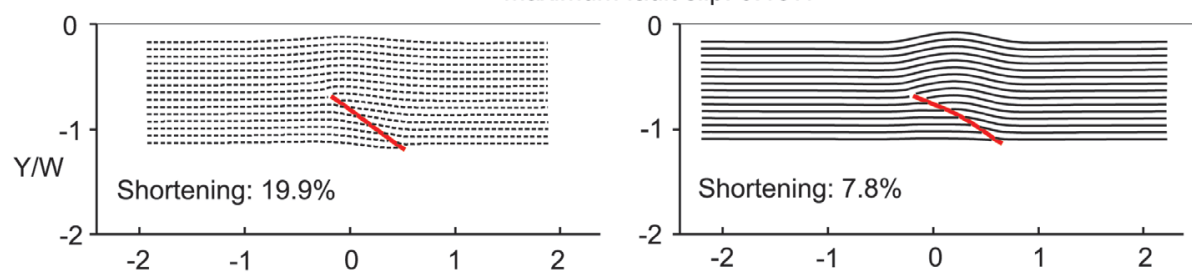

Shortening: $7.8 \%$
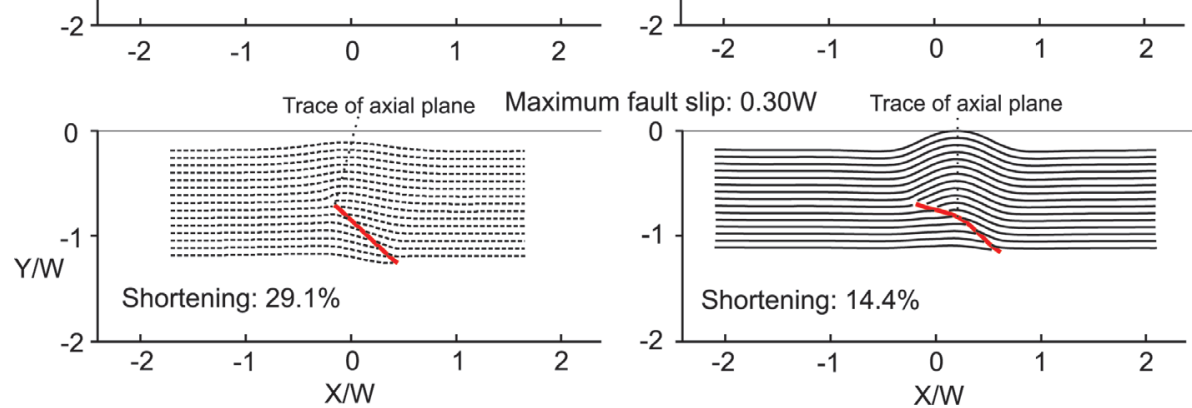

Fig. 5. Models of a fault embedded in layers. $W$ is initial fault width. Fault dips $25^{\circ}$ at onset. (a) Fault-cored passive fold (layer interfaces are bonded). (b) Fault-cored buckle fold (Layers slip freely at contacts). (Color online only) 
differences between the passive and buckle folds in Fig. 5 are as follows: (1) the amplitudes of the fault-cored buckle fold grow faster than the amplitudes of the fault-cored passive fold. (2) After the maximum fault slip of $0.30 \mathrm{~W}$, the fold in the fault-cored buckle fold is more highly localized with steeper limb dips than the fault-cored passive fold. (3) The buckle fold is nearly concentric while the passive is somewhat asymmetric with a short forelimb dipping to the left. (4) The fold wavelength, measured as the distance between synclinal hinges on the flanks of the anticline, is shorter in the fault-cored buckle fold than in the fault-cored passive fold. (5) The ratio of the average folded interface amplitude to the maximum slip on the fault in the fault-cored buckle fold is 0.6 while the ratio for the fault-cored passive fold is 0.3. Thus, with the same amount of fault slip, the buckle fold amplitude is about 2 times the passive fold amplitude.

\subsubsection{Ramp Anticline}

The setting for this case is fault-bend folding over a flat-ramp-flat fault embedded in the layering. We show three stages in which the total displacement on the semiinfinite dislocation is $0 W, 0.38 W$, and $0.71 W$, where $W$ is the width of the ramp in Fig. 6. The ramp initially dips $25^{\circ}$. An anticline forms above the ramp in both the bonded and freely sliding cases. However, the geometry of the anticlines is distinctly different, namely: (1) like the previous two models, the amplitudes of ramp-cored buckle fold grow faster than the amplitudes of the ramp-cored passive fold. (2) After total hanging wall displacement of $0.71 \mathrm{~W}$, the folded interfaces in the ramp-cored buckle fold are box-like with two localized shear bands with opposite-facing limbs above the upper and lower ends of the ramp. The localized folding of the forelimb and backlimb with nearly uniform limb dips closely resembles the geometry produced in passive layers by the ramp fold model for anisotropic materials (e.g., Erickson et al. 2001). The folding somewhat resembles the angular fault-bend kinematic model fold (e.g., Suppe 1983), however, the relatively flat anticline crest is tilted in this model in contrast with the horizontal crest assumed in the fault-bend fold kinematic model. In comparison, the rampcored passive fold is broad and gentle. (3) The fold wavelength, measured as the distance between synclinal hinges on the anticline flanks, is similar in the two models. (4) The ratio of the average amplitude of folded interfaces to the maximum slip on the fault in the ramp-cored buckle fold is 0.27 while the ratio in the ramp-cored passive fold is 0.15 . Thus, with the same amount of fault slip, the fold amplitude in the ramp-cored buckle fold is nearly twice the amplitude of the fold in the ramp-cored passive fold.

\subsection{Influence of Buckling on Fold Form}

We demonstrated that fault-cored fold forms can be sig- nificantly influenced by fault geometry, properties of layer contacts, and loading conditions. Hereafter in this section, we will use the setting of a fault in basement underlying sedimentary layering to examine the effects of two factors on fold forms: the free ground surface and the thickness of layers.

\subsubsection{Ground Surface Effect}

We plot the fold amplitudes of the folded interfaces in Fig. 4 with a maximum fault slip of $0.3 \mathrm{~W}$ in Fig. 7 in addition to the folded interface amplitudes of a buckle fold and a fault-cored passive fold in a full-space medium with the same setting. The amplitude at a layer horizon is measured as the vertical difference between the anticline crest and the lower synclinal hinge on one of the anticline flanks. Figure 7 shows the amplitudes of the fault-cored buckle folds are larger than the fault-cored passive folds. Furthermore, the amplitude of the two fault-cored passive folds decreases upwards whereas the amplitude of the fault-cored buckle fold near the ground surface increases upward away from the fault. This is clearly an effect of the free surface. Figure 7 shows that the amplitude of a fault-cored buckle fold far below the free surface does not steadily increase upwards from the fault.

\subsubsection{Layer Thickness Effect}

A series of fault-cored folds with different layer thicknesses is shown in Fig. 8. The thicknesses of mechanical layers vary but the total thickness of the entire stack of layers is the same in each fold. All folds in Fig. 8 are formed by shortening until fault slip reaches a maximum of $0.18 \mathrm{~W}$. The ratio of their amplitudes to the amplitudes of fault-cored passive fold with the same fault slip is plotted as a function of the number of layer interfaces in Fig. 9a. Fold amplitude increases as the number of layers increases. The amplitude of the top layer interface of the fault-cored buckle fold with 25 layer interfaces is nearly 6 times the amplitude of the fault-cored passive fold under the same amount of shortening. The wavelength of the fault-cored buckle folds is plotted as a function of the number of layer interfaces in Fig. 9b. The wavelength decreases as the number of layer interfaces increases. The wavelength of the fault-cored buckle fold with 10 or more layers is less than half the wavelength of the fault-cored passive fold (i.e., $n=0$ ).

\section{COALINGA AND KETTLEMAN HILLS ANTICLINES, CALIFORNIA}

We now examine actively growing anticlines at Coalinga and Kettleman Hills in Central California for which we have data relating slip on the fault to the growth of the folds. Surface displacements were recorded from moderate earthquakes in 1983 and 1985 on the faults underlying the Coalinga and Kettleman Hills anticlines. 


\subsection{Setting}

A $110 \mathrm{~km}$-long chain of Quaternary fault-cored en echelon anticlines is located in Central California approximately $30 \mathrm{~km}$ east of the San Andreas fault and on the western edge of the San Joaquin Valley (Fig. 1). The anticlinal axes of the folds trend nearly parallel to the San Andreas fault. During 1982 - 1985, three moderate earthquakes $\left(5.4 \leq \mathrm{M}_{\mathrm{w}} \leq 6.5\right)$ occurred along this chain of anticlines on reverse faults underlying the folds (e.g., Stein and Ekström 1992). Figure 1 shows profiles across the chain of folds constructed from well and seismic reflection data (Meltzer 1989; Wentworth and Zoback 1989; Stein and Ekström 1992).

As discussed in the introduction a series of papers on the earthquakes (King and Stein 1983; Stein and King 1984; Ekström et al. 1992) suggested that the Kettlemen Hills Anticlines grow primarily by slip on the underlying fault during repeated large earthquakes like the 1982 - 1985 sequence. In contrast, the peak of the vertical displacement pattern for the 1985 Kettleman Hills earthquake is offset

(a) Ramp-cored passive folding

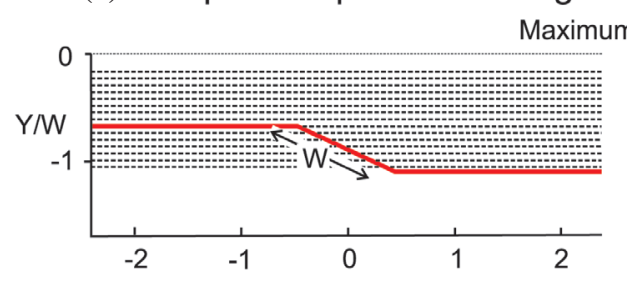

(b) Ramp-cored buckle folding

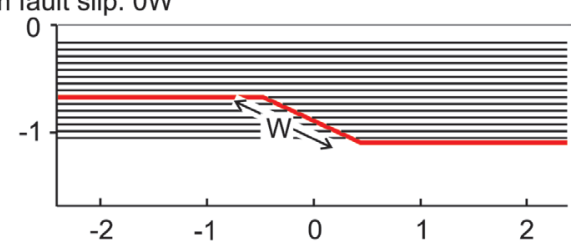

Maximum fault slip: $0.38 \mathrm{~W}$
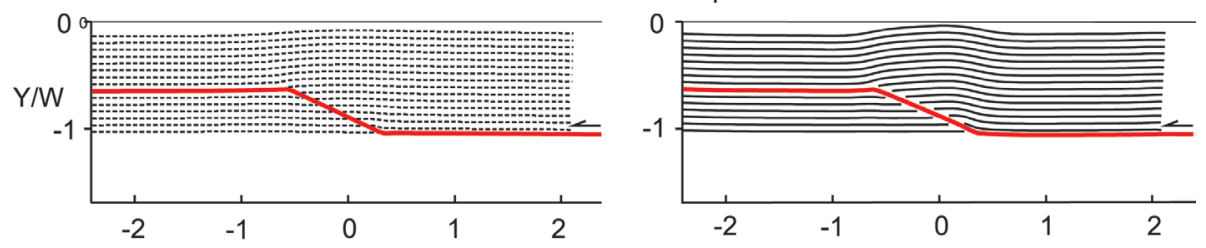

Maximum fault slip: $0.71 \mathrm{~W}$
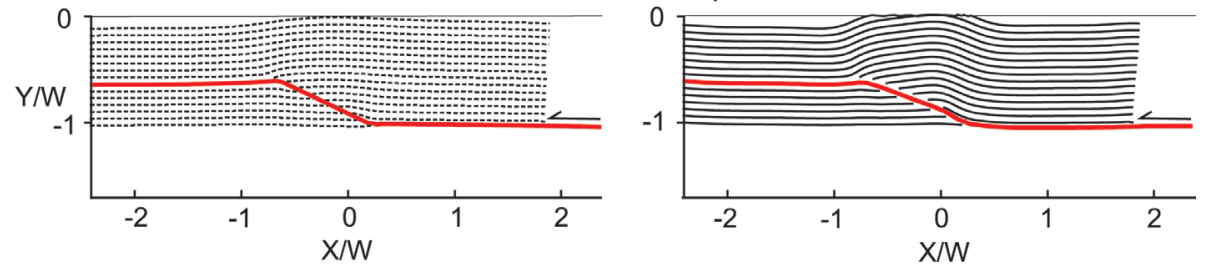

Fig. 6. Models of a ramp anticline. $W$ is the width of ramp. Ramp initially dips $25^{\circ}$. The maximum fault slip is the amount of slip applied to the detachment at the far right side of the model domain. (a) Ramp-cored passive fold (layer interfaces are bonded). (b) Ramp-cored buckle fold (layers slip freely at contacts). (Color online only)

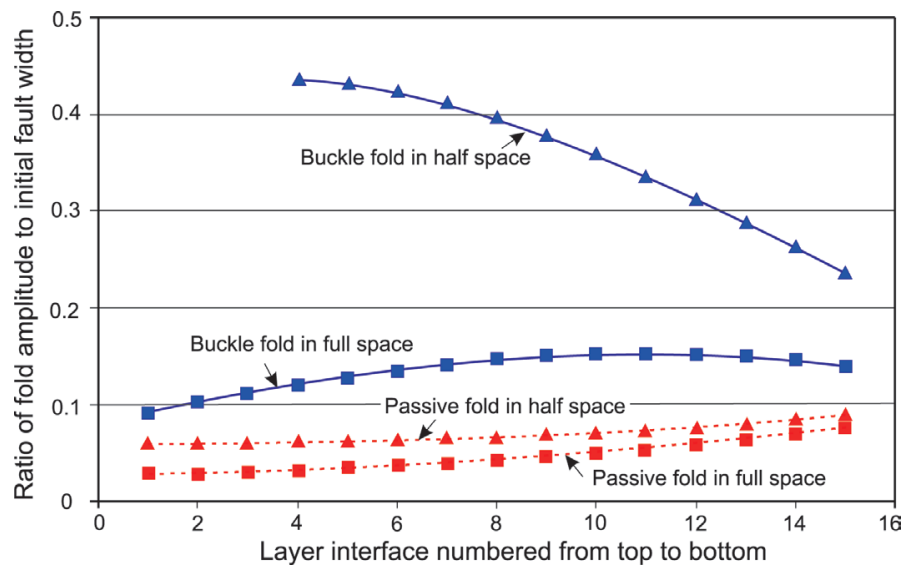

Fig. 7. Comparison of fold amplitudes in Fig. 5 and fault-cored passive and buckle fold amplitudes in a full space (no free surface). (Color online only) 

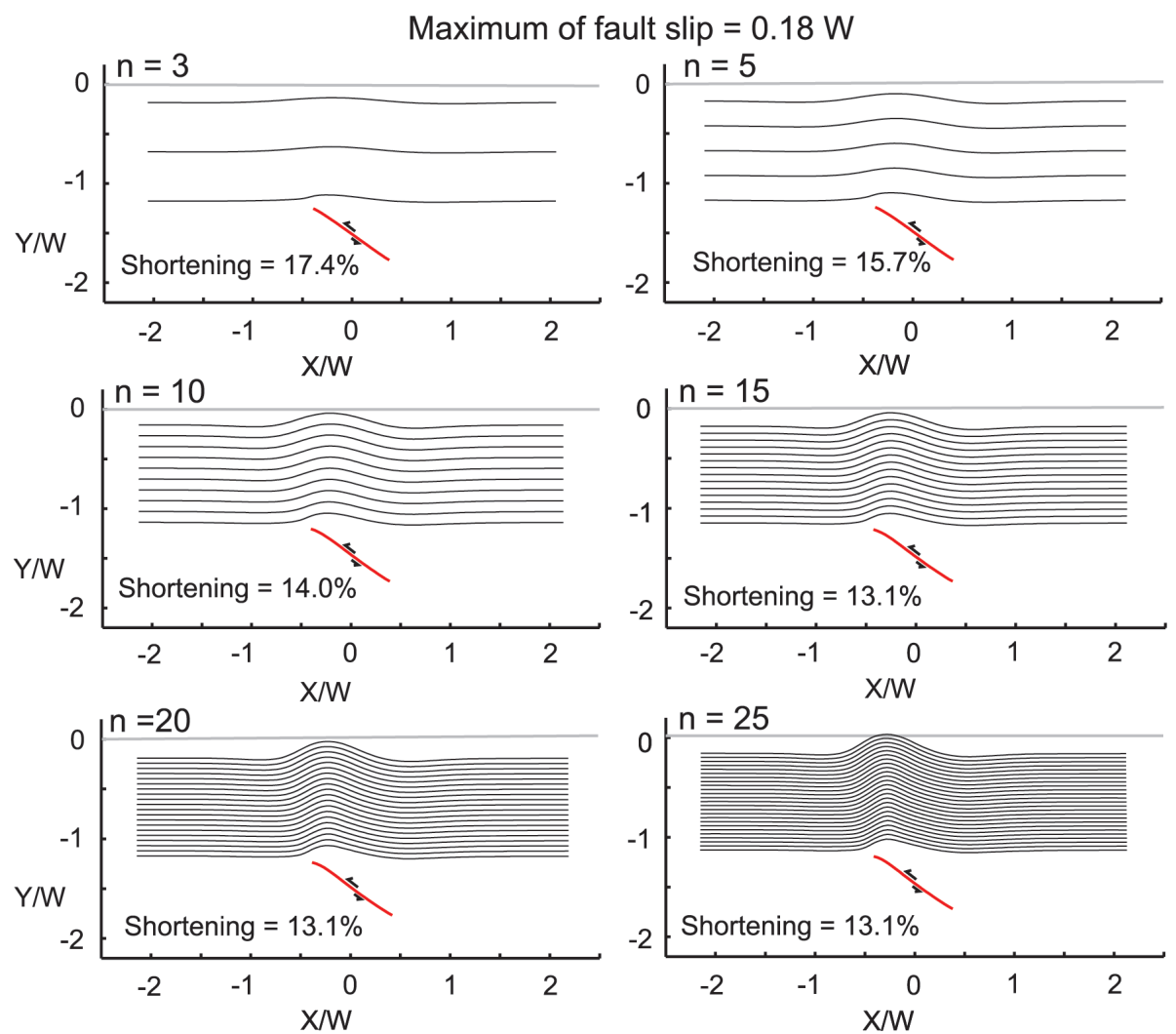

Fig. 8. Models of a fault embedded in stacks of mechanical layers with different layer thicknesses. The faults in all models have the same maximum fault slip of $0.18 W . W$ is initial fault width. $n$ is number of layer interfaces. (Color online only)
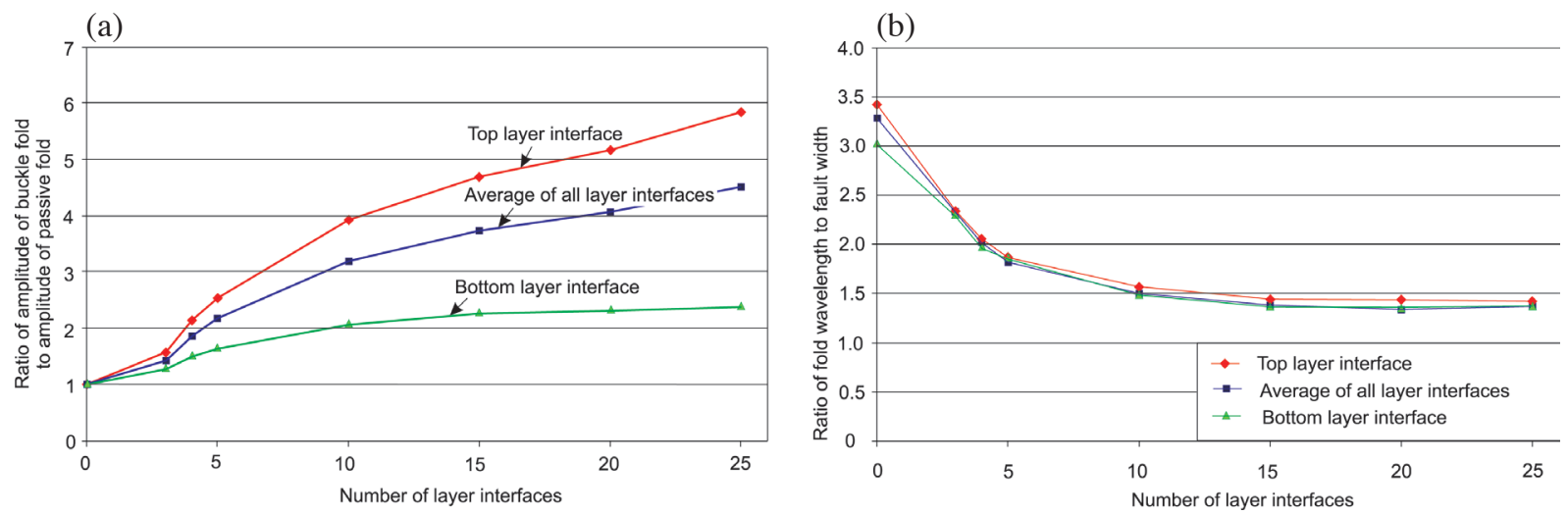

Fig. 9. Comparisons of fold features with different numbers of layer interfaces. (a) Ratio of fault-cored buckle fold amplitudes in Fig. 8 to fold amplitude in passive markers under the same amount of shortening plotted with number of layer interfaces. (b) Fold wavelengths from Fig. 8 normalized by the initial fault width plotted with number of layer interfaces. (Color online only)

about $3 \mathrm{~km}$ NE of the fold axis of the Kettleman Hills North Dome. Figure 10 shows the vertical displacement contours from an elastic dislocation model with uniform slip that best reproduces the vertical displacement measurements during the 1985 earthquake (Stein and Ekström 1992). Stein and Ekström (1992) suggested that the North Dome probably grew as a result of repeated earthquakes on a dipping fault under the fold, similar to the fault that slipped in the 1985 earthquake. However, that fault has perhaps begun migrating to the northeast, generating uplift to the northeast of the anticline during the 1985 earthquake.

We will demonstrate that an alternative explanation is that the 1985 earthquake may very well be typical of earthquakes on the major fault underlying the anticline, but the coseismic deformation pattern does not match the fold geometry because the fold did not grow solely as a consequence of 
slip on the underlying fault.

\subsection{Mechanical Analysis}

Results from our analyses of fault-cored buckle folds with an embedded shallowly dipping fault (Fig. 5) show that the anticline crest is well behind the fault tip, whereas models of the same type but with passive layering show the fold crest above the fault tip. Figure 10b shows the fold in a mechanically layered medium along with vertical displacement pattern at the ground surface due to slip on the buried reverse fault. The relationship between the location of the peak coseismic uplift and the axial trace of the anticline is similar to that observed from the 1985 Kettleman Hills earthquake. The peak coseismic uplift is shifted to the front limb of the anticline, not centered on the anticline. We therefore suggest that this result indicates that the Kettleman Hills North Dome likely formed as a buckle fold overlying a reverse fault, similar to those produced in our model. The shape of the vertical displacement pattern due to earthquakes on the underlying reverse fault does not directly reflect the shape of the anticline because the anticline grows by combined fault slip and layer buckling mechanisms.

The subsurface shape of the Kettleman Hills South Dome (Fig. 1c) is further evidence that buckling contributes significantly to the growth of the anticline. Figure $11 \mathrm{com}-$ pares models of folds produced by shortening of a medium with a $45^{\circ}$ dipping reverse fault with either passive markers or mechanical layers. The geometry of the fold produced by the model with mechanical layers is similar to the Kettleman Hills South Dome. The seismic profile and the model both show a slightly asymmetric fold localized above the dipping reverse fault. The fold produced in a homogeneous medium with passive markers clearly does not resemble the actual fold and the modeled amplitude is too small.

This analysis of the Kettleman Hills Anticlines is revealing, but is far from complete. To determine the extent to which the fold has recently grown between large

(a)
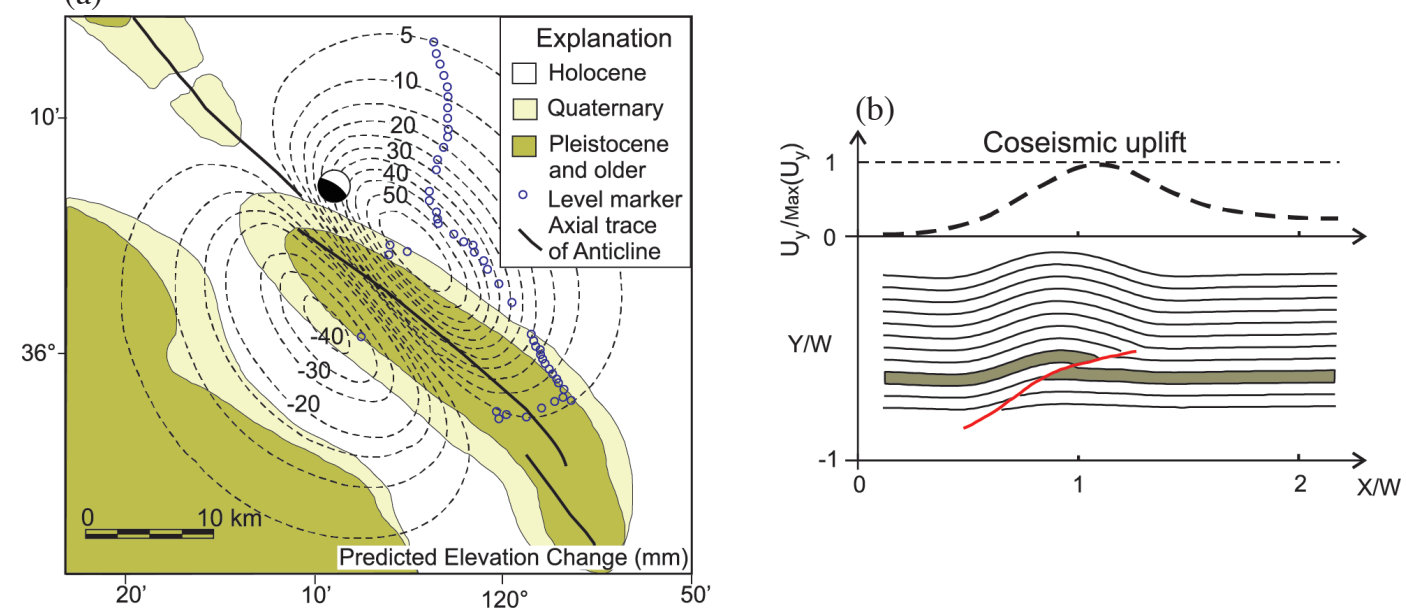

Fig. 10. Predicted elevation change of 1985 Kettleman Hills earthquake. (a) Vertical deformation field predicted from a rectangular dislocation with reverse-slip by Ekström et al. (1992). (b) Model of a fault embedded in mechanical layers like in Fig. 6 . $W$ is initial width of the fault and $U_{y}$ is vertical displacement. The plot of coseismic uplift attributed to only fault slip in the upper part of (b) is centered above the forelimb of Kettleman Hills Anticline, much like the observed pattern in (a).
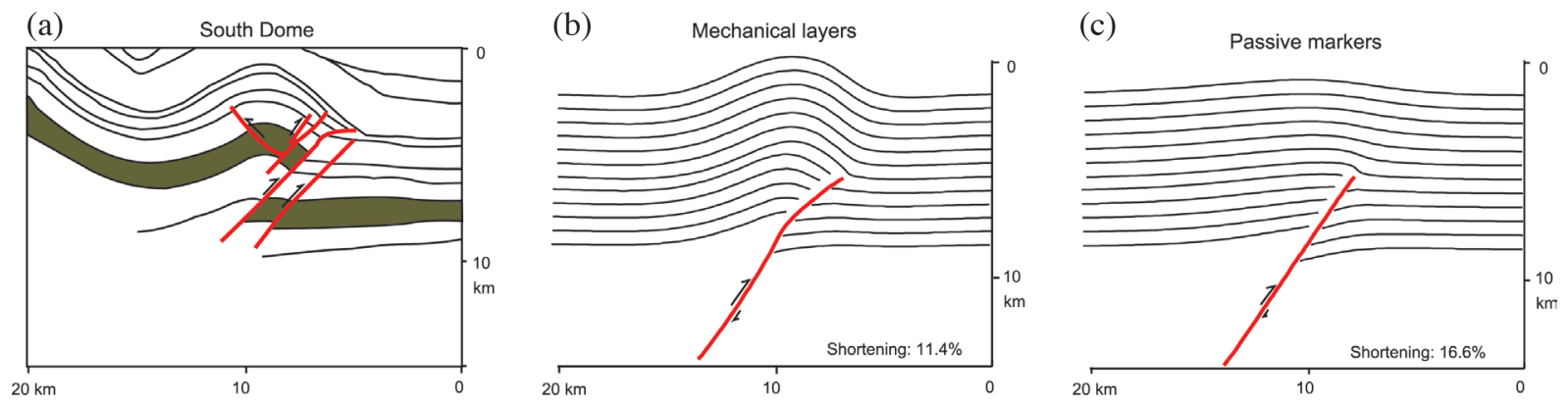

Fig. 11. Comparison of South Dome anticline and simulations. (a) Profile of Kettleman Hills South Dome (profile in Fig. 11c). (b) Result from mechanical layer model. (c) Result from passive marker model. The anticline in (b) resembles the South Dome anticline better than the anticline in (c). (Color online only) 
earthquakes, either by slip on the underlying fault or buckling, one would want to examine interseismic data showing the deformation pattern. One would also want to examine geomorphic evidence for Holocene deformation of the fold in order to determine the longer-term deformation history. Acquisition and analysis of such data in future work would likely yield valuable insight about the fold growth processes at Kettleman Hills.

\section{CONCLUSIONS}

We have constructed boundary element models with a medium containing a fault and elastic layers subjected to layer-parallel shortening to demonstrate the influence of buckling on fault-cored fold growth. Free slip is assumed on the fault and layer contacts. We compare folding simulations in the mechanically layered elastic medium with passive marker folding in a non-layered medium. Given the same amount of far-field shortening for both conditions, the mechanically layered medium produces more highly localized folds with higher amplitude and shorter wavelength.

The horizontal shortening that causes a fault in an anticline core to slip can cause significant amplification of the fold by buckling of the strata. Under the conditions considered in this paper, the contribution to fold growth by slip on the underlying fault alone is only about $20-50 \%$ of the total growth. Therefore, studies that seek to estimate fault slip from fold geometry by assuming the fold is built by slip on the fault alone could significantly overestimate the amount of fault slip.

At Kettleman Hills Anticline in Central California, published seismic profiles show the subsurface fault and fold geometry and slip on the fault can be inferred from coseismic uplift data from the 1985 Kettleman Hills earthquake. We show that the general features of the fold form are consistent with the BEM model that incorporates buckling of layers over a dipping, blind reverse fault. Furthermore, the general coseismic uplift pattern centered over the anticline forelimb is predicted by the model.

Acknowledgements We thank Arvid M. Johnson for providing his analytical viscous folding theory results to verify our BEM method and many useful suggestions that helped improve this article.

\section{REFERENCES}

Allmendinger, R. W. and J. H. Shaw, 2000: Estimation of fault propagation distance from fold shape: Implications for earthquake hazard assessment. Geology, 28, 1099-1102, doi: 10.1130/0091-7613(2000) 28<1099:EOFPDF>2.0.CO;2. [Link]

Berger, P. and A. M. Johnson, 1980: First-order analysis of deformation of a thrust sheet moving over a ramp.
Tectonophysics, 70, T9-T24, doi: 10.1016/0040-1951(80)90276-0. [Link]

Berger, P. and A. M. Johnson, 1982: Folding of passive layers and forms of minor structures near terminations of blind thrust faults - application to the central Appalachian blind thrust. J. Struct. Geol., 4, 343-353, doi: 10.1016/0191-8141(82)90018-9. [Link]

Biot, M. A., 1961: Theory of folding of stratified viscoelastic media and its implications in tectonics and orogenesis. Geol.Soc.Am.Bull.,72, 1595-1620, doi: 10.1130/00167606(1961)72[1595:TOFOSV]2.0.CO;2. [Link]

Biot, M. A., 1963: Exact theory of buckling of a thick slab. Appl.Sci.Res., 12, 183-198, doi: 10.1007/BF03184639. [Link]

Biot, M. A., 1964: Theory of internal buckling of a confined multilayered structure. Geol. Soc. Am. Bull., 75, 563568, doi: 10.1130/0016-7606(1964)75[563:TOIBOA]2 .0.CO;2. [Link]

Cardozo, N., 2008: Trishear in 3D. Algorithms, implementation, and limitations. J. Struct. Geol., 30, 327-340, doi: 10.1016/j.jsg.2007.12.003. [Link]

Cardozo, N., K. Bhalla, A. T. Zehnder, and R. W. Allmendinger, 2003: Mechanical models of fault propagation folds and comparison to the trishear kinematic model. J. Struct. Geol., 25, 1-18, doi: 10.1016/S0191-8141(02)00013-5. [Link]

Cardozo, N., R. W. Allmendinger, and J. K. Morgan, 2005: Influence of mechanical stratigraphy and initial stress state on the formation of two fault propagation folds. J. Struct. Geol., 27, 1954-1972, doi: 10.1016/j. jsg.2005.06.003. [Link]

Chapple, W. M., 1969: Fold shape and rheology: The folding of an isolated viscous-plastic layer. Tectonophysics, 7, 97-116, doi: 10.1016/0040-1951(69)90001-8. [Link]

Chester, J. S. and F. M. Chester, 1990: Fault-propagation folds above thrusts with constant dip. J. Struct. Geol., 12, 903-910, doi: 10.1016/0191-8141(90)90063-5. [Link]

Cooke, M. L. and D. D. Pollard, 1997: Bedding-plane slip in initial stages of fault-related folding. J. Struct. Geol., 19, 567-581, doi: 10.1016/S0191-8141(96)00097-1. [Link]

Crouch, S. L. and A. M. Starfield, 1983: Boundary element methods in solid mechanics. J. Appl. Mech., 50, 704705, doi: 10.1115/1.3167130. [Link]

Ekström, G., R. S. Stein, J. P. Eaton, and D. Eberhart-Phillips, 1992: Seismicity and Geometry of a 110-km-long blind thrust fault 1 . The 1985 Kettleman Hills, California, earthquake. J. Geophys. Res., 97, 4843-4864, doi: 10.1029/91JB02925. [Link]

Elliott, D., 1976: The motion of thrust sheets. J. Geophys. Res., 81, 949-963, doi: 10.1029/JB081i005p00949. [Link]

Erickson, S. G. and W. R. Jamison, 1995: Viscous-plastic finite-element models of fault-bend folds. J. Struct. 
Geol., 17, 561-573, doi: 10.1016/0191-8141(94)00082B. [Link]

Erickson, S. G., L. M. Strayer, and J. Suppe, 2001: Initiation and reactivation of faults during movement over a thrust-fault ramp: Numerical mechanical models. $J$. Struct. Geol., 23, 11-23, doi: 10.1016/S0191-8141(00)00074-2. [Link]

Erslev, E. A., 1991: Trishear fault-propagation folding. Geology, 19, 617-620, doi: 10.1130/0091-7613(1991)019<0617:TFPF>2.3.CO;2. [Link]

Erslev, E. A. and K. R. Mayborn, 1997: Multiple geometries and modes of fault-propagation folding in the Canadian thrust belt. J. Struct. Geol., 19, 321-335, doi: 10.1016/ S0191-8141(97)83027-1. [Link]

Finch, E., S. Hardy, and R. Gawthorpe, 2003: Discrete element modelling of contractional fault-propagation folding above rigid basement fault blocks. J. Struct. Geol., 25, 515-528, doi: 10.1016/S0191-8141(02)00053-6. [Link]

Fletcher, R.C., 1977: Folding of a single viscous layer: Exact infinitesimal-amplitude solution. Tectonophysics, 39, 593-606, doi: 10.1016/0040-1951(77)90155-X. [Link]

Grant, L. B., K. J. Mueller, E. M. Gath, H. Cheng, R. L. Edwards, R. Munro, and G. L. Kennedy, 1999: Late Quaternary uplift and earthquake potential of the San Joaquin Hills, southern Los Angeles basin, California. Geology, 27, 1031-1034, doi: 10.1130/0091-7613(1999)027<1031:LQUAEP>2.3.CO;2. [Link]

Ishiyama, T., K. Mueller, M. Togo, A. Okada, and K. Takemura, 2004: Geomorphology, kinematic history, and earthquake behavior of the active Kuwana wedge thrust anticline, central Japan. J. Geophys. Res., 109, B12408, doi: 10.1029/2003JB002547. [Link]

Johnson, A. M., 1977: Styles of Folding, Elsever Publishing Company, New York, 426 pp.

Johnson, A.M. and P. Berger, 1989: Kinematics of fault-bend folding. Eng. Geol., 27, 181-200, doi: 10.1016/00137952(89)90033-1. [Link]

Johnson, A. M. and V. J. Pfaff, 1989: Parallel, similar and constrained folds. Eng. Geol., 27, 115-180, doi: 10.1016/0013-7952(89)90032-X. [Link]

Johnson, A. M. and R. C. Fletcher, 1994: Folding of Viscous Layers, Columbia University Press, $461 \mathrm{pp}$.

Johnson, K. M. and A. M. Johnson, 2002: Mechanical analysis of the geometry of forced-folds. J. Struct. Geol., 24, 401-410, doi: 10.1016/S0191-8141(01)00085-2. [Link]

Kilsdonk, B. and R. C. Fletcher, 1989: An analytical model of hanging-wall and footwall deformation at ramps on normal and thrust faults. Tectonophysics, 163, 153168, doi: 10.1016/0040-1951(89)90123-6. [Link]

King, G. and R. Stein, 1983: Surface folding, river terrace deformation rate and earthquake repeat time in a reverse faulting environment: The Coalinga, California, earthquake of May 1983. Calif. Div. Mines Geol., 66,
165-176.

Langdon, T. G., 1970: Grain boundary sliding as a deformation mechanism during creep. Philos. Mag., 22, 689700, doi: 10.1080/14786437008220939. [Link]

Le Béon, M., J. Suppe, M. K. Jaiswal, Y. G. Chen, and M. E. Ustaszewski, 2014: Deciphering cumulative fault slip vectors from fold scarps: Relationships between long-term and coseismic deformations in central Western Taiwan. J. Geophys. Res., 119, 5943-5978, doi: 10.1002/2013JB010794. [Link]

Mancktelow, N. S., 1999: Finite-element modelling of singlelayer folding in elasto-viscous materials: The effect of initial perturbation geometry. J. Struct. Geol., 21, 161177, doi: 10.1016/S0191-8141(98)00102-3. [Link]

Martel, S. J. and J. R. Muller, 2000: A two-dimensional boundary element method for calculating elastic gravitational stresses in slopes. Pure Appl. Geophys., 157, 989-1007, doi: 10.1007/s000240050014. [Link]

McClay, K. R., 1977: Pressure solution and Coble creep in rocks and minerals: A review. J. Geol. Soc., 134, 5770, doi: 10.1144/gsjgs.134.1.0057. [Link]

Meglis, I. L., R. E. Gagnon, and R. P. Young, 1995: Microcracking during stress-relief of polycrystalline ice formed at high pressure. Geophys. Res. Lett., 22, 2207 2210, doi: 10.1029/95GL02007. [Link]

Meltzer, A. S., 1989: Crustal structure and tectonic evolution: Central California. Ph.D. Thesis, Rice University, Texas, 95-167.

Myers, D. J., J. L. Nabelek, and R. S. Yeats, 2003: Dislocation modeling of blind thrusts in the eastern Los Angeles basin, California. J. Geophys. Res., 108, doi: 10.1029/2002JB002150. [Link]

Mynatt, I., G. E. Hilley, and D. D. Pollard, 2007: Inferring fault characteristics using fold geometry constrained by Airborne Laser Swath Mapping at Raplee Ridge, Utah. Geophys. Res. Lett., 34, L16315, doi: 10.1029/2007GL030548. [Link]

Niño, F., H. Philip, and J. Chéry, 1998: The role of bedparallel slip in the formation of blind thrust faults. $J$. Struct. Geol., 20, 503-516, doi: 10.1016/S0191-8141(97)00102-8. [Link]

Patton, T. L. and R. C. Fletcher, 1995: Mathematical blockmotion model for deformation of a layer above a buried fault of arbitrary dip and sense of slip. J. Struct. Geol., 17, 1455-1472, doi: 10.1016/0191-8141(95)00034-B. [Link]

Reches, Z. and A. M. Johnson, 1978: Development of monoclines: Part II. Theoretical analysis of monoclines. Geol. Soc. Am. Mem., 151, 273-312, doi: 10.1130/ MEM151-p273. [Link]

Rich, J. L., 1934: Mechanics of low-angle overthrust faulting as illustrated by Cumberland thrust block, Virginia, Kentucky, and Tennessee. AAPG Bull., 18, 1584-1596, doi: 10.1306/3D932C94-16B1-11D7-8645000102C1865D. 
[Link]

Sanford, A. R., 1959: Analytical and experimental study of simple geologic structures. Geol. Soc. Am. Bull., 70, 19-52, doi: 10.1130/0016-7606(1959)70[19:AAESOS 12.0.CO;2. [Link]

Shackleton, J. R. and M. L. Cooke, 2007: Is plane strain a valid assumption in non-cylindrical fault-cored folds? J. Struct. Geol., 29, 1229-1240, doi: 10.1016/j. jsg.2007.03.011. [Link]

Shaw, J. H. and P. M. Shearer, 1999: An elusive blind-thrust fault beneath metropolitan Los Angeles. Science, 283, 1516-1518, doi: 10.1126/science.283.5407.1516. [Link]

Shaw, J. H., A. Plesch, J. F. Dolan, T. L. Pratt, and P. Fiore, 2002: Puente Hills blind-thrust system, Los Angeles, California. Bull. Seismol. Soc. Am., 92, 2946-2960, doi: 10.1785/0120010291. [Link]

Sibson, R. H., 1986: Earthquakes and rock deformation in crustal fault zones. Annu. Rev. Earth Planet. Sci., 14, 149-175, doi: 10.1146/annurev .earth.14.1.149. [Link]

Smart, K. J., D. A. Ferrill, and A. P. Morris, 2009: Impact of interlayer slip on fracture prediction from geomechanical models of fault-related folds. AAPG Bull., 93, 1447-1458, doi: 10.1306/05110909034. [Link]

Stein, R. S. and G. C. P. King, 1984: Seismic potential revealed by surface folding: 1983 Coalinga, California, earthquake. Science, 224, 869-872, doi: 10.1126/science.224.4651.869. [Link]

Stein, R.S. and G. Ekström, 1992: Seismicity and Geometry of a $110-\mathrm{km}$-long blind thrust fault 2. Synthesis of the 1982-1985 California Earthquake Sequence. J. Geophys. Res., 97, 4865-4883, doi: 10.1029/91JB02847. [Link]

Strayer, L. M. and J. Suppe, 2002: Out-of-plane motion of a thrust sheet during along-strike propagation of a thrust ramp: A distinct-element approach. J. Struct. Geol., 24, 637-650, doi: 10.1016/S0191-8141(01)00115-8. [Link]

Suppe, J., 1983: Geometry and kinematics of fault-bend folding. Am. J. Sci., 283, 684-721, doi: 10.2475/ ajs.283.7.684. [Link]

Suppe, J. and D. A. Medwedeff, 1994: Fault-propagation folding. Geol. Soc. Am. Abstr. Programs, 16, 670.

Taboada, A., J. C. Bousquet, and H. Philip, 1993: Coseismic elastic models of folds above blind thrusts in the Betic Cordilleras (Spain) and evaluation of seismic hazard. Tectonophysics, 220, 223-241, doi: 10.1016/00401951(93)90233-A. [Link]

Wentworth, C. M. and M. D. Zoback, 1989: The style of Late Cenozoic deformation at the eastern front of the California Coast Ranges. Tectonics, 8, 237-246, doi: 10.1029/TC008i002p00237. [Link]

Wiltschko, D. V., 1979: A mechanical model for thrust sheet deformation at a ramp. J. Geophys. Res., 84, 1091-1104, doi: 10.1029/JB084iB03p01091. [Link]

Yamashita, T. and K. Ojima, 1968: Deformation twin tips with flat surfaces in pure iron crystals. J. Electron Microsc., 17, 301-308

\section{APPENDIX A1: COMPARISON OF ANALYTICAL AND BEM SOLUTIONS FOR AMPLIFICATION FACTOR}

To verify that our BEM program is reliable for solving folding problems, we compare amplification factors computed with the analytical solution (Johnson and Pfaff 1989) and with the boundary-element solution. It was not intuitively obvious that a folding model (BEM) consisting of layers modeled with small boundary elements at their interfaces would be equivalent (or nearly so) to analytical folding theory (Johnson and Fletcher 1994). The amplification factor is related exponentially to the amplitude growth rate of a fold. The larger the amplification factor, the faster the fold amplitude grows. The amplification factor for a given set of conditions is a function of the wavelength to thickness ratio of a single layer within a multilayer. Thus, Fig. A1 shows plots of amplification factors calculated using the two methods for two, four and ten identical layers. The peak in each curve has coordinates of the maximum amplification factor and the dominant wavelength. The dominant wavelength is the wavelength that will grow the fastest.

By comparing amplification factors calculated with the BEM and the analytical model, we see that the results are very similar (Fig. A1). The similarity shows that the two models, derived in quite different ways, are almost certainly addressing the same mechanical problem. Thus we can depend on the BEM model to solve some folding problems such as those addressed in this paper that would be very difficult with the analytical folding theory.

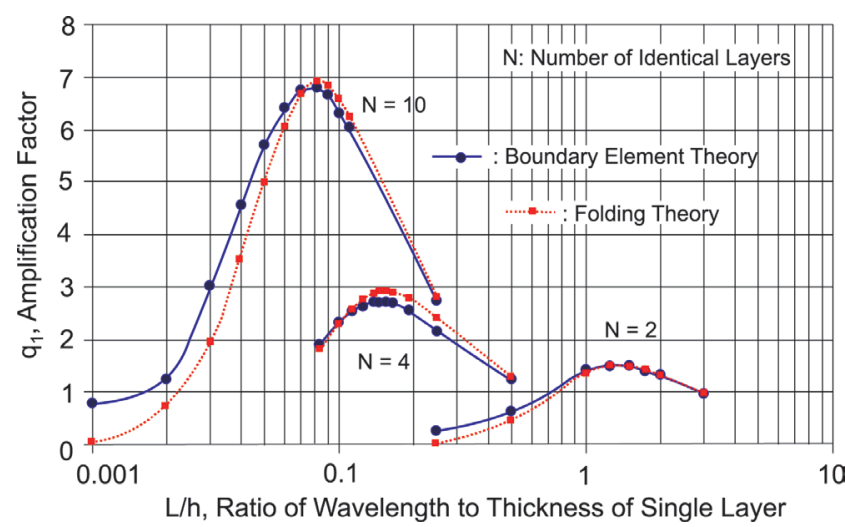

Fig. A1. Plots of amplification factor of periodic folds using analytical viscous folding theory and boundary element theory. (Color online only) 